\title{
Engineering Practices of Determining Transmission Capacity and Delay of Interconnecting Line Taking into Account its Configuration and Cost
}

\author{
Igor Parkhomey ${ }^{1}$, Juliy Boiko ${ }^{2}$, Nataliia Tsopa ${ }^{3}$, Oleksander Eromenko ${ }^{4}$ \\ ${ }^{1,3}$ Department of Technical Cybernetics, National Technical University of Ukraine "Igor Sikorsky Kyiv Polytechnic \\ Institute", Ukraine \\ ${ }^{2}$ Department of Telecommunications and Radio Engineering, Khmelnytsky National University, Ukraine \\ ${ }^{4}$ Department of Physics and Electrical Engineering, Khmelnytsky National University, Ukraine
}

\begin{tabular}{l} 
Article Info \\
\hline Article history: \\
Received Sep 21, 2019 \\
Revised Sep 21, 2020 \\
Accepted Sep 23, 2020 \\
\hline Keywords: \\
Engineering optimization \\
practice \\
Transmission capacity \\
Delay time \\
Transit delay
\end{tabular}

Article Info

Revised Sep 21, 2020

Accepted Sep 23, 2020

\section{Keywords:}

practice

Delay time

\begin{abstract}
This article contains information on engineering practice of determining transmission capacity of computer network line. The article presents a variant of engineering synthesis of computer network, which is a combined process of mathematical and heuristic methods combining. The engineering synthesis is offered as vector and global, because it must result in network development, optimal in terms of its practical use. All the significant network quality indicators, including economic and practical, are taken into consideration. In case of engineering synthesis, it is not possible that only one quality indicator is significant: there are always at least two significant indicators - a cost and an indicator that characterizes the main effect that is achieved in case of network use (efficacy). If at least one of the quality indicators significant for practical use is not taken into account, such network cannot be considered optimal. Computer network synthesis usually consists of structure synthesis, parameters optimization and discrete network selection. If network topology is maintained unchanged, it is possible to formulate an optimization task for line transmission capacity. The solution of transmission capacity task, which is constantly changing, may be chosen as a starting point for the selection of discrete indicator of transmission capacity.
\end{abstract}

Copyright @ 2020 Institute of Advanced Engineering and Science. All rights reserved.

Corresponding Author:

Juliy Boiko,

Department of Telecommunications and Radio Engineering,

Khmelnytsky National University,

11, Instytuts'ka str., Khmelnitsky, 29016, Ukraine.

Email: boiko_julius@ukr.net

\section{INTRODUCTION}

One of the most important tasks in communication network optimization is the estimation of main indicators, which characterize its quality. They can be called external or baseline parameters. Let's mark these parameters as $a_{1}, a_{2}, .$. , an. They include, for example, transferred information delay $P_{e r}$, error possibility during discrete messages transmission, $\Delta t_{m}$ mean time to failure, and $C$ cost. Based on engineering task conditions, the values of some $a_{1}, a_{2}, \ldots$, an parameters must be fixed, while others can be modified within certain limitations during the engineering process. Let say that $a_{1}, a_{2}, .$. , an is an external modified parameter [1-3].

Let's consider the impact of monotonous increase of one of these parameters on the network quality, for example, an under other equal conditions, i.e. permanent network operating conditions and permanent values of all other parameters. It is obvious that only one of the following cases is possible, when ai increase causes system quality to:

- improve;

- worsen; 
- remain unchanged;

- change not monotonously.

At this stage of engineering it is not possible to establish the dependency character of network quality from $a_{i}$ parameter values $[4,5]$.

It is obvious that in the third case parameter ai does not affect the network quality and may be even omitted in the process of engineering. In the future, if during monotonous increase parameter $a_{i}$ gains the value of the first or second case, this parameter is called network quality indicator and is marked as $k_{i}$. In all the other cases ai does not belong to the class of quality indicators and is called simply modified network parameter [4].

Therefore, $k_{i}(i=1,2, \ldots, m)$ network quality indicator is the numerical network characteristic which is related to its quality be means of strict monotonous dependency - the more (the less) the $k_{i}$ value is, the better the network is in case of other equal conditions.

In general, we may say, even without analyzing the network's activity, which external variable parameters in case of pre-set baseline data can be considered as quality indicators (for example, error possibility, delay, transmission capacity, reliability, and cost). On the contrary, the number of transit nodes and the number of network users do not belong to quality indicators [5-7].

In order to define if this $a_{i}$ indicator can be considered a quality indicator, the impact of this indicator on the system quality is taken into consideration in case of other equal conditions, in particular, in saving all the other network indicators [6].

Even in case of such indicator as $C$ cost, we are not able to affirm that "the less the cost is, the better the system is", as a cost decrease may cause the deterioration of such most important quality indicators as error possibility and delay. The introduction of $k_{1}, \ldots, k_{m}$ quality indicators allows the exclusion of undoubtedly worse network creation variants during the process of engineering, due to this fact it is not only possible to avoid acceptance of undoubtedly worse solution, but also frequently to facilitate and speed up the finding of optimal (better) solution $[1,6]$.

\section{RESEARCH METHOD}

The cumulative amount of all $D=\left\{D_{1}, \ldots, D_{n}\right\}$ baseline data can be divided into three subgroups:

- total amount of several $Y=\left\{Y_{1}, \ldots, Y_{n}\right\}$ conditions;

- total amount of $O_{s}=\left\{O_{s 1}, \ldots, O_{s Q}\right\}$ limitations per structure and planned network parameters;

- composition of the total amount (vectors) of $K=\left\langle k_{1}, \ldots, k_{m}>\right.$ network quality indicators;

- total amount of $O_{k}=\left\{O_{K 1}, \ldots, O_{K R}\right\}$ limitations, which are imposed on quality indicators.

The $S$ network creation variant, which complies with the total amount of $S\{Y, O\}$ baseline data, shall be called acceptable. In this case not only one acceptable network may exist, but some set of acceptable Mn networks. The acceptable network, which complies with the total amount of $O k$ limitations, is called strictly acceptable. In other words, strictly acceptable network is the network, which complies with all the total amount of $D=\left\{Y, O_{s}, O_{k}\right\}$. Not only one strictly acceptable network may exist, but some set of strictly acceptable $M_{m}$ networks. Of all the strictly acceptable networks Sopt the network, which has the best (out of the pre-established) vector value of $K$ quality indicators, is considered to be optimal (the best). Hence, in order to choose the optimal network, an advantage criterion is pre-selected (justified), and, as a rule, one $K$ vector value is considered to be better than its other value.

Not long ago, the search of non-optional optimal network was not in priority: the engineering task was considered to be successfully solved, if any strictly acceptable network was found (designed). However, in recent years the task to design not only strictly acceptable, but also optimal networks becomes more relevant. This is due to the fact that every year the requirements to informational communication networks increase. That's why not only the compliance with the $O_{k}$ baseline requirements, which are applied to the system quality indicators, is significant, but also the improvement of these indicators.

Frequently the search of optimal network is called network synthesis $[8,9]$.

Synthesis task may be defined as: to find such network, which complies with the total amount of $\left\{Y, O_{s}, C_{k}, O_{\mathrm{k}}\right\}$ baseline data and has the best $K=\left\langle k_{1}, \ldots, k_{m}\right\rangle$ quality indicators values, which are treated as preestablished advantage criterion (network optimality criterion).

Let's define a combining process of mathematical and heuristic methods as engineering synthesis.

Complex system synthesis usually has to be vector.

Vector synthesis is the synthesis which takes into account several quality indicators, i.e. by the vector value of $K=\left\langle k_{1}, \ldots, k_{m}\right\rangle$ quality indicators. Vector synthesis is also defined as vector optimization, optimization by vector criterion or multi-criteria optimization. In contrast to this, synthesis, which is performed with the use of single $(m=1)$ quality indicator, is called scalar.

Global synthesis is the synthesis which takes into account all the significant quality indicators, including economical and practical. It the synthesis takes into account not all the significant quality 
indicators, it is called partial. It is easy to assure that engineering synthesis has to be vector or global due to the following considerations. Engineering synthesis results in network development, which is considered to be optimal in terms of its practical use. Therefore, in the process of this synthesis all the significant quality indicators are taken into consideration. If at least one of the significant quality indicators in terms of practical use is not taken into account, such network cannot be considered optimal.

Hence, an engineering synthesis always has to be global. In case of engineering synthesis, it is not possible that only one quality indicator is significant: there are always at least two significant indicators $-C$ cost and an indicator, which characterizes the main effect that is achieved in case of network use (efficacy). In practice the amount of significant quality indicators is more than two. This results in the fact that global synthesis, and therefore the engineering synthesis, is always vector [7-11].

In case of mathematical synthesis on the contrary to engineering synthesis, in order to make mathematical task solution possible or to simplify it, sometimes some significant indicators are not taken into account, for example, economical (quantitative) and practical indicators. Therefore, mathematical synthesis can be both global and partial.

Synthesis of communication system or devices usually contains solution of such main tasks:

- optimal network (system) structure synthesis;

- selection of optimal network parameter values, for example, transferred information delay, authenticity, minimal amount of managing information in the management system, which provides the preestablished accuracy of managed network parameters etc., or parameter optimization;

- selection of optimal variant of network (system) creation from the definite amount of $N$ of completely defined $S_{1}, S_{2}, . ., S_{N}$ variants or discrete network (system) selection.

Therefore, the synthesis of informational communication network usually consists of structure synthesis, optimization parameters and discrete network selection.

If network topology is maintained unchanged, it is possible to formulate an optimization task for system transmission capacity and line transmission capacity [12, 13].

There are some of analytical methods to solve this problem. But there are some of heuristic methods, which are more effective as shown $[14,15]$.

We will imply the system transmission capacity, in which the call flow enters, such us the magnitude of the load that can enter this system while support a set quality of service, that is, a set amount of loss. Some does not imply by the system transmission capacity like the received traffic, which can be served with the required quality, but the traffic passed through into the system. The numerical difference between these definitions is insignificant in the field low-losses. In the region of large admissible losses, transmission capacity at the first definition can considerably increase than by the second. From the position of the convenience of calculations, the first definition is more convenient [16].

The system transmission capacity depends on many factors. It is a function of the capacity of the beams, of losses, of the class of calls flow, depends on the structure of the switching system, on the scheme of combining the outputs of switching devices, and also is a function of the law of time distribution and quality of service.

Each of these factors has a different impact on transmission capacity, and may, by-turn, have a rather complex structure, which is characterized by a number of parameters. The rate of loss can be set not only as a whole, but also separately for each direction of transmission or for certain categories of subscribers. An increase the allowable loss rate leads results to an increase in capacity because of a deterioration of the communication quality [17-19].

The call flow can be a simple flow. In this case it is characterized by one number - the mathematical expectation of the calls number per unit time. Or it could be the Palm`s flow, which is characterized by the distribution function of intervals between calls, the number of call sources and the flow`s parameter which is generated by each source. The distribution of service time can be exponential distribution of a constant value, the Erlang's law.

Service quality can be defined in different ways. In a waiting system, calls can be serviced in the order of receipt, in a random order, in the inverse order of receipt, with different types of priorities. In a lossy system, the quality of service can consist in successive passing of a call from one stage to another, if there is a conditional search through several stages, at the existence of a certain cyclic or random order of fill of lines. Quality of service may vary with the passage of time depending on the circumstances [20-22].

The structure of the dial-up system is also characterized by a large number of parameters: the number of cascades, the connectivity, the construction and methods of communication between different switchboards, blockade, etc.

In this regard, it is looks much simpler for line. Too high line transmission capacity requires additional costs, and too low line transmission capacity leads to overloads and message delay. But in practice, line transmission capacity cannot be modified without any limitations. It is selected from the 
discrete set. However, the solution of the task with the transmission capacity, which constantly changes, provides interesting results and can serve as a starting point for the selection of transmission capacity discrete value.

The simplest ratio for $(D)$ full cost and line characteristics is linear and looks like [7]:

$$
D=D_{e}+10 \cdot \lambda_{i} \cdot d_{i}
$$

where: $D_{e}-$ is the money amount left after the payment for net line transmission capacity;

$d_{i}-$ cost coefficient for $i-$ that line;

$\lambda_{i}-$ line traffic intensity.

$d_{i}$ values are not identical, as lines have different length.

In order to get a simple expression for $(T)$ delay time due to $C_{i}$ transmission capacity, it is necessary to perform a number of simplified actions: to accept that message lengths have exponential allocation, and the moments of their occurrence in each node create Poisson point process [23]. These assumptions make all the tasks related to queues independent. Not taking into account such a big simplification of the task, therefore the defined $T$ is agreed with the result of a more accurate modelling in a vast range of traffic intensity.

The average delay for $i-$ that $\left(T_{i}\right)$ line is considered to be the time of waiting in queue:

$$
T_{i}=\frac{1}{\mu C_{i}-\lambda_{i}},
$$

where: $C_{i}-$ the transmission capacity of $i-$ that line, $\lambda_{i}-$ line package traffic intensity, and $(1 / \mu)-$ average package length in bits.

In order to gain the average value of all the processes with queues, $T_{i}$ values are narrowed using the $\left(\lambda_{i} / \gamma\right)$ ratio, where $\gamma$ is a cumulative speed of message receipt. Therefore, the cumulative delay of all the packages within 1 second of $\sum_{i}\left(\lambda_{i} \cdot T_{i}\right)$ network operation is divided into the total quantity of packages, which are transmitted through the network in 1 second.

$(T)$ - average delay in this case is defined by the expression:

$$
T=\sum_{i} \frac{\lambda_{i}}{\gamma}\left(\frac{1}{\mu C_{i}-\lambda_{j}}\right),
$$

a similar average value for the number of this package transmission through some line during its transit movements is defined through $\bar{n}$ and by the ratio:

$$
\bar{n}=\sum_{i} \frac{\lambda_{i}}{\gamma}
$$

The expression for $T$ can be clarified in case of adding the members, which take into account message processing time and line transmission time.

Transmission capacities, which minimize the delay in case of permanent line cost are defined by method of Lagrange multipliers and are pre-established by equations:

$$
C_{i}=\frac{\lambda_{i}}{\mu}+\frac{D_{e}}{d_{i}} \frac{\sqrt{\lambda_{i} d_{i}}}{\sum \sqrt{\lambda_{i} d_{i}}},
$$

where $D_{e}$ is defined by expression:

$$
D_{e}=D-\frac{\lambda_{i} d_{i}}{\mu},
$$

$\left(\lambda_{i} / \mu\right)$ value is the line transmission capacity in relation to traffic transfer through $i$ - that line in saturation mode (net transmission capacity), in this case the right item in the right part (5) can be considered as 
additional transmission capacity, added by this line. Net transmission capacity leads to full $\Sigma\left(\lambda_{i} d_{i}\right) / \mu$ cost in a way that $D_{e}$ in equation (1) is the money amount left after the payment for net transmission capacity.

Equation (4) is called "Square root law in case of additional transmission capacity allocation", since it shows that the given transmission capacity must be allocated proportionally to the square root from the traffic intensity in the line. The value of effective transit delay in case of transmission through lines with transmission capacity, which is pre-established by the equation (5), is defined as:

$$
T_{o p t}=\frac{\bar{n}}{\mu D_{e}}\left(\sum_{i} \sqrt{\frac{\lambda_{i} d_{i}}{\lambda}}\right)^{2}
$$

In this expression $\lambda$ defines the amount of traffic intensity in all the lines. Since $\bar{n}$ denotes the average number of transit lines, which pass through the package, this expression simply and clearly depends from package transmission time and the amount of such transmissions. Average transit time, as can be observed, is inversely proportional to $D_{e}$ money amount left after the payment for net transmission capacities of all lines.

\section{RESULTS AND DISCUSSION}

Consider an example of network which topology is shown in Fig. 1. It consists of 6 lines with different physical standards. Each segment of this network has its own length and characteristics. Transmission capacities and delay were calculated (formulas 4 and 6 accordingly) for each network segment. The results are presented in the form of a diagram in Fig. 2 and Fig. 3.

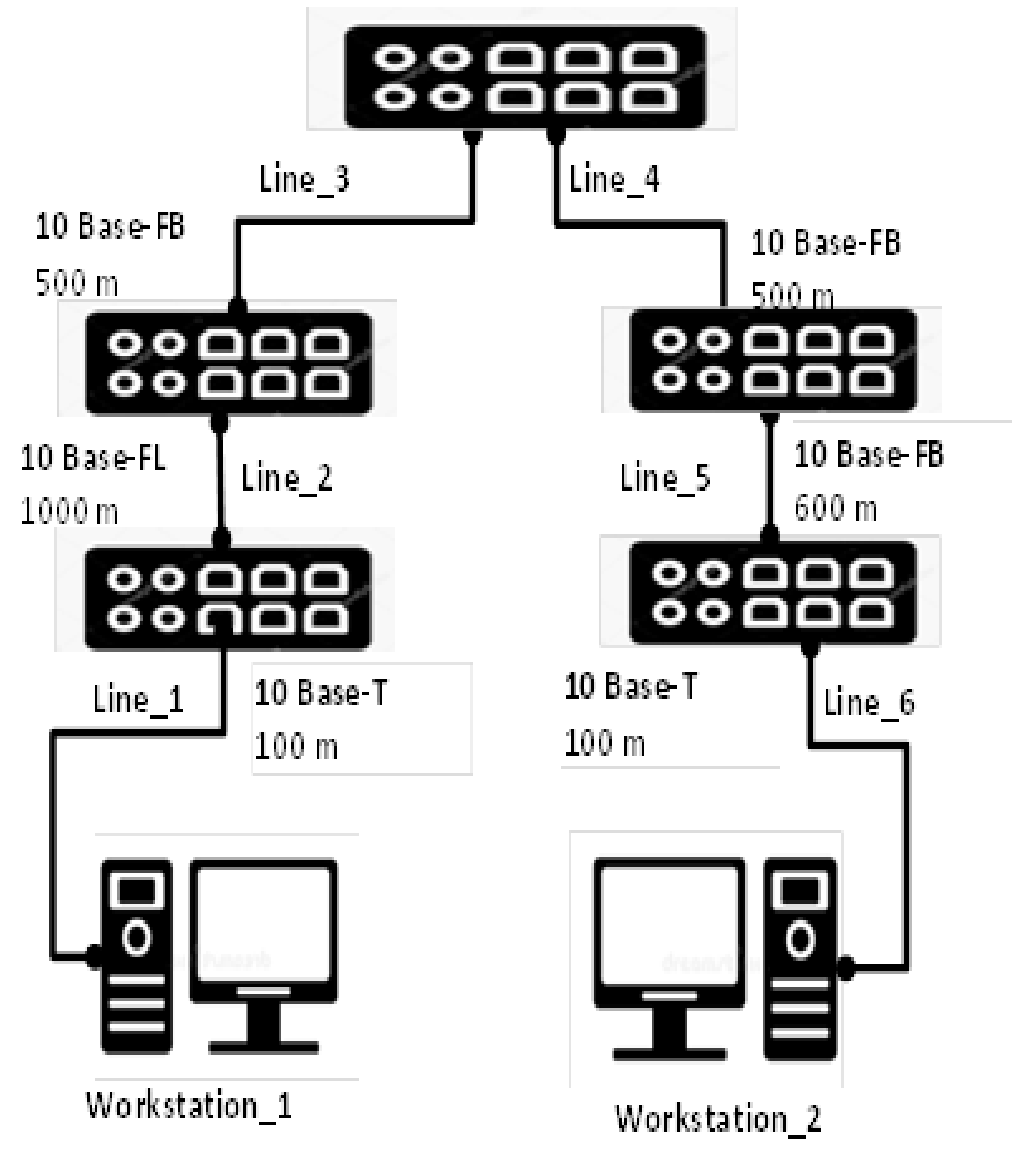

Figure 1. The example of a network which consisting segments of different physical standards

These diagrams allow analyzing the influence of the considered characteristics of each segment on the network as a whole. As can be seen from the diagram in Fig. 1, line 1 has the greatest effect on the transmission capacities of the entire network (about 30\%). Also, it has the lowest delay value - 5\%. Line 5 has the worst estimated figures. It has the greatest delay value in relation to the delay along the entire line. 
Let's explore a practical implementation of two more engineering practices of communication line (channel) selection by pre-established parameters, which allows the simplification and facilitation of configuration selection task and interconnecting line (channel) type between different network lines [24, 25]. They are the following practices:

- the practice of interconnecting line assessment taking into account its configuration and cost;

- the practice of interconnecting line selection taking into account its transmission capacity and cost.

\section{The transmission capacity of $i$ - that line}

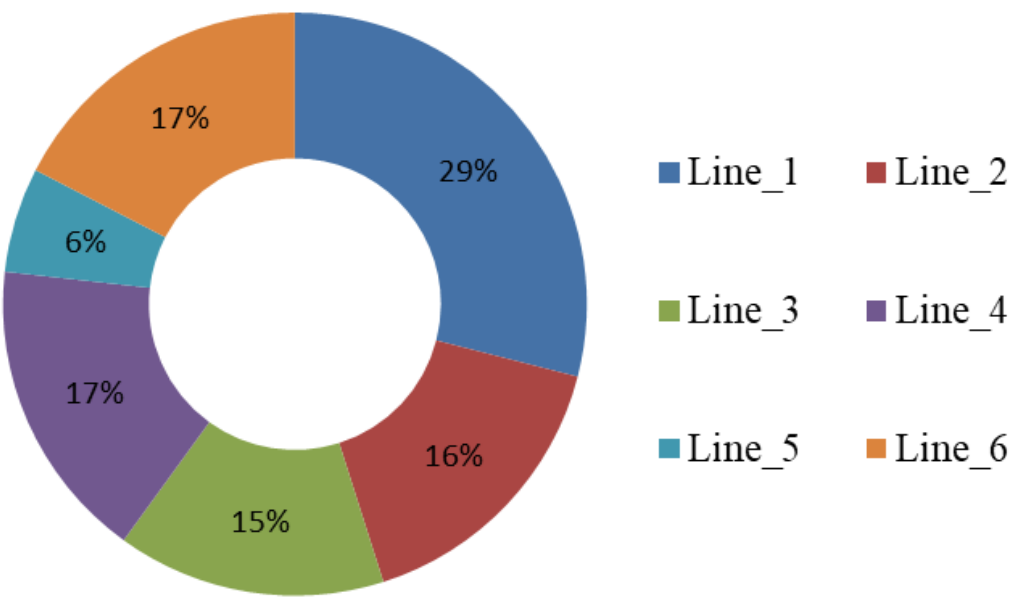

Figure 2. Diagram of calculated values for the transmission capacity of each line of discussed network

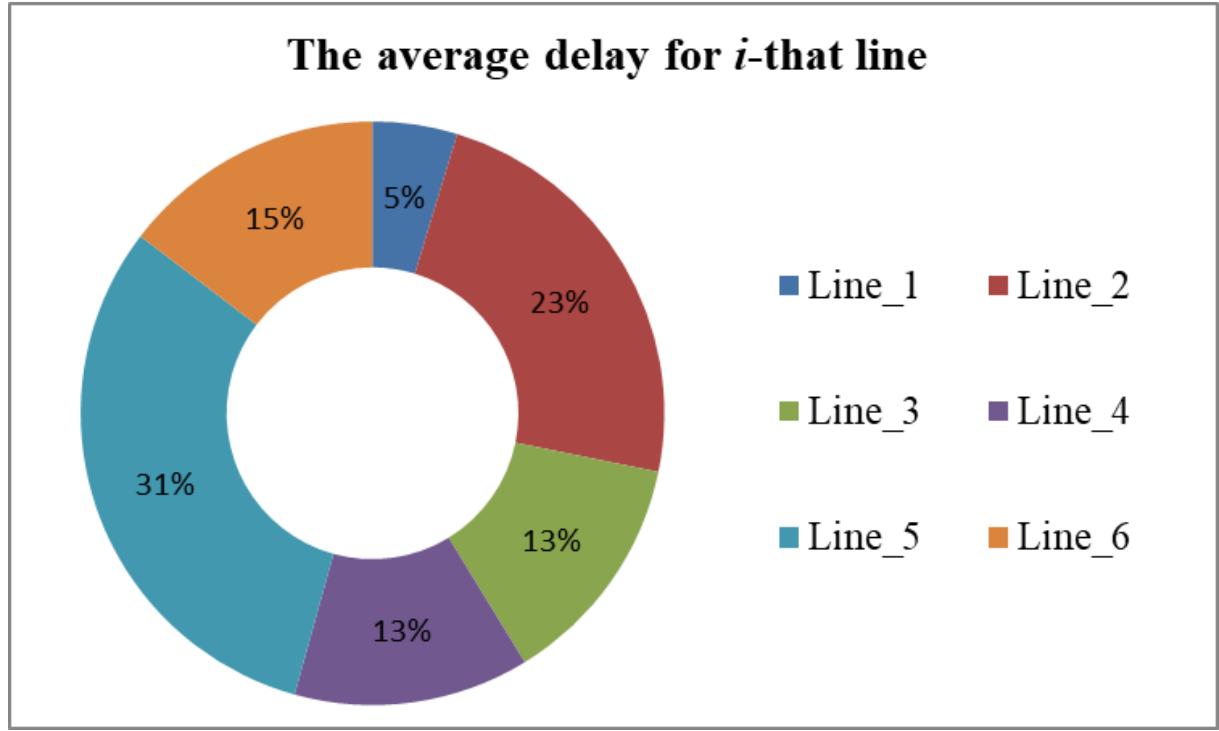

Figure 3. Diagram of calculated values for the average delay of each line of discussed network

The first practice provides an opportunity to select a line by pre-established values of its cost and message transmission authenticity on the basis of the input of its configuration data, separate line components cost and error possibility, which are caused by these components.

As known, almost any interconnecting line information can be presented in the form of cumulative amount of consistent and parallel connections of separate sections of interconnecting line, which have its own cost and possibility of error occurrence.

This forms the base of the given practice.

Let's consider the program listing of the proposed practice.

The program is written in Prolog language [26]. In the first program section (domains) the proposed user "language" is defined. 


$\begin{array}{ll}\text { cable } & \text { single line element (cable) } \\ \operatorname{seq}(a, b) & \text { consistent connection of some a and b structures } \\ \operatorname{par}(a, b) & \text { parallel connection of two structures }\end{array}$

With the launch of the program, at first the existing resources database is downloaded, model.dat, which has, for example, a following form:

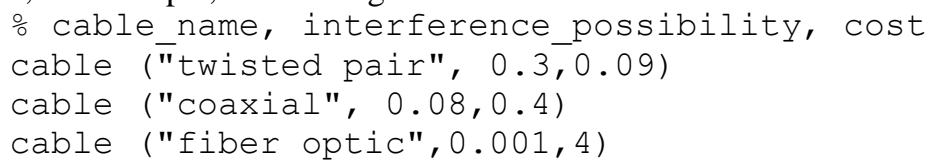

Then the program, using an integrated semantic analyzer Prolog'a, enables the user to input by means of keyboard a line structure (par(par(cable, cable),cable),cable).

Further the process predicate, depending on user input, activates the install predicate (output of all possible solutions) or list filter predicate (output of solutions with filtration according to established criteria). Both install and list filter predicates launch solution predicate until Prolog-system, based on existing facts, still can find solutions and show them on the screen.

Let's consider solution predicate structure.

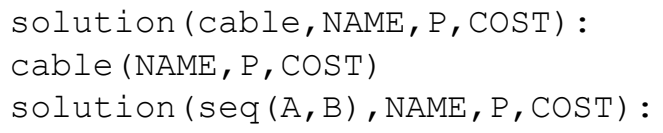

then:

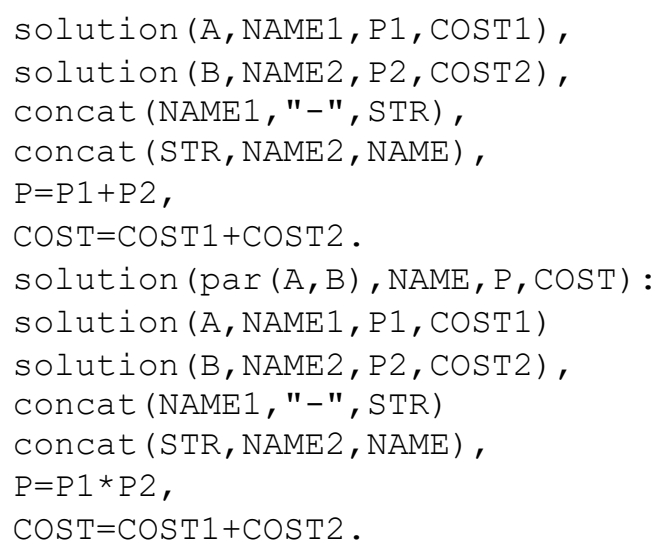

in case of a singe cable,

find the appropriate value in the database

in case of consistent A and B connection,

1. find solution for $A$;

2. find solution for $\mathrm{B}$;

3. sum up the possibility of failure;

4. sum up the costs.

in case of parallel A and B connection,

1. find solution for $A$;

2. find solution for $B$;

3. multiply error possibility

4. sum up the cost.

The second practice provides an opportunity to gain cost configuration and line transmission capacity according to the established information volume, that is transmitted, and acceptable authentic information.

This practice is implemented by means of a program, which provides automatic generation of all the possible configuration variants by several criteria (cost, transmission capacity and authenticity).

The program is written in Prolog language.

Straight after the launch, the program establishes the initial value of "variables" and downloads the database of assistant materials.

Further the program shows the user a request for the necessary line (channel) parameters - volume of data array, maximal acceptable time of its transmission, necessary authenticity. After that a smodel.rep file is opened for the output of the reporting information on all of the found solutions, and gensol predicate is launched as many times, as it is possible in case of the established system parameters and current database contents. After gensol predicate launch, several solution variants are shown on the screen which comply the most with the following criteria:

1. Information array transmission in the established time, by the volume closest to the necessary.

2. The least amount of separate communication lines.

3. The smallest cost of communication line rent.

Gensol predicate, during its launch, activates solution predicate and then outputs the found solutions into the report file.

Let's consider solution predicate structure of this program in detail.

solution $(\mathrm{X}, \mathrm{X}, \mathrm{A}, \ldots, 0,0):-\mathrm{A}<=0$, !. 
if previously found configuration can perform the transmission in the necessary amount of time or less, then all

solution (INL, OUTL, TOTAL, TIME, SEND, OUTPR): in the other case:

database;

channel (CHN,BPS, PRI), 1 select any connection line from the

MIDLIST $=[$ CHN $\mid$ INL $]$,

$D E L T A=T O T A L-T I M E * B P S, \quad 2$ 2. How many more bits need to be

transmitted

solution (MIDLIST, OUTL, DELTA, TIME, X, PR2,

3. calculate the configuration of the rest of the system.

TIME * BPS $+\mathrm{X}>=$ TOTAL,

$\mathrm{OUTPR}=\mathrm{PR} 1+\mathrm{PR} 2$,

$\mathrm{SEND}=\mathrm{TIME} * \mathrm{BPS}+\mathrm{X}$.

$\mathrm{P}=\mathrm{P} 1 * \mathrm{P} 2$

\section{CONCLUSION}

The proposed engineering practices provides an opportunity to perform a previous calculation of line transmission capacity during the transmission via computer networks lines, which can help perform primary network optimization and achieve more detailed calculations.

Given practices take into account all the network-significant quality indicators, including economic and practical. A ratio for the full cost and line characteristics, which are linear, are presented. A formula of average delay for $i$-the line, which is defined by the time of waiting in queue, is introduced. Based on the average delay, a similar average value for the number of this package passing through some line during its transit movements. Transmission capacities, which minimize the delay time in case of permanent line cost and which are calculated by the method of Lagrange multipliers, are estimated. The transmission capacity of the line in relation to traffic transmission through $i$ - the line in the saturation mode (net transmission capacity) is determined. A formula for the calculation of the effective transit delay value during transmission through the lines with determined transmission capacity is presented.

\section{REFERENCES}

[1] M. Chiang, S. H. Low, A. R. Calderbank and J. C. Doyle, "Layering as Optimization Decomposition: A Mathematical Theory of Network Architectures," Proceedings of the IEEE, vol. 95, iss. 1, pp. 2007.

[2] X. Liu, et al, "Analysis of communication requirements for typical distribution network business," in IEEE 3rd Information Technology, Networking, Electronic and Automation Control Conference, 2019. ITNEC 2019, pp. 1-4.

[3] X. Hu, et al., "Risk modeling and optimization approach for system protection communication networks," in IEEE/IFIP Network Operations and Management Symposium, 2018. NOMS 2018, 2018, pp.1-4.

[4] E. Seve, et al., "Learning process for reducing uncertainties on network parameters and design margins," IEEE/OSA Journal of Optical Communications and Networking, vol.10, iss. 2, pp. A298 - A306. 2018.

[5] O. Kennedy, et al., "Comparative analysis of the performance of various active queue management techniques to varying wireless network conditions," International Journal of Electrical and Computer Engineering, vol.9 no. 1, pp. 359-367. 2019.

[6] F. H. Tavakoli, et al., "Determining optimal location and capacity of DG units based on system uncertainties and optimal congestion management in transmission network," in Iranian Conference on Electrical Engineering, 2017. ICEE 2017, pp. 1-4.

[7] M. K. Mahmood, F. M. M. Al-Naima, and Z. Zaidan, "Reliability Assessment of the Iraqi National Communication Network," Indonesian Journal of Electrical Engineering and Informatics, vol. 6, no. 4, pp.448-457. 2018.

[8] G. Wang, R. Gu and Y. Ji, "Resource allocation optimization for time and wavelength division multiplexing passive optical network enabled mobile fronthaul with bitrate-variable compressed common public radio interface," IEEE/OSA Journal of Optical Communications and Networking, vol. 8, iss. 6, pp. 417 - 426. 2016.

[9] P. Zhao, L. Feng, P.Yu, W. Li and X. Qiu, "A fairness resource allocation algorithm for coverage and capacity optimization in wireless self-organized network," China Communications, vol. 15, iss. 1, pp. 10-24. 2018.

[10] I. M. Ismail, and N. N. Agwu," Influence of Heuristic Functions on Real-Time Heuristic Search Methods," in 14th IEEE International Conference on Electronics Computer and Computation, 2018. ICECCO 2018, pp. 1-4.

[11] J. Verma, S. Gupta, P. Manhas and Vasudha Arora, "Target Network Selection Algorithm based on Required Dwell Time Estimation," Indonesian Journal of Electrical Engineering and Informatics, vol. 6, no. 2, pp. 172-182. 2018.

[12] Z. Zali, et al., "A controller-based architecture for information centric network construction and topology management," China Communications, vol. 15, iss. 7, pp. 131-145. 2018. 
[13] J. Boiko, I. Kovtun and S. Petrashchuk, "Productivity of telecommunication systems with modified signal-code constructions", in Proceedings 4th IEEE International Scientific-Practical Conference Problems of Infocommunications. Science and Technology 2017. PIC S\&T 2017, pp. 173-178.

[14] S. G. Glisic, Self-Organizing Networks, 3rd ed. IEEE Books for Purchase : Wiley Telecom, 2016.

[15] B. Nemeth, et al., "The limits of architectural abstraction in network function virtualization," in IFIP/IEEE International Symposium on Integrated Network Management, 2015. IM 2015, pp. 1-6. 2015.

[16] T. H. Q. Minh, "Hybrid Time-Power Switching Protocol of Energy Harvesting Bidirectional Relaying Network: Throughput and Ergodic Capacity Analysis," Telkomnika(Telecommunication Computing Electronics and Control), vol. 16, no 5, pp. 1947-1956. 2018.

[17] I. Parkhomey, et al., "Assessment of quality indicators of the automatic control system influence of accident interference, " Telkomnika (Telecommunication Computing Electronics and Control), vol. 18, no. 4, pp. 2070-2079, Aug. 2020

[18] A. Khafidin, T. Andrasto and S. Suryono, "Implementation flow control to improve quality of service on computer networks," Indonesian Journal of Electrical Engineering and Computer Science, vol.16 no 3, pp. 1474-1481. 2019.

[19] J. Boiko and O. Eromenko, "Signal Processing in Telecommunications with Forward Correction of Errors. Indonesian Journal of Electrical Engineering and Computer Science, vol.11 no 3, pp. 868-877. 2018.

[20] I.B. Paraschuk, "System formation algorithm of communication network quality factors using artificial neural networks," in 1st IEEE International Conference on Circuits and Systems for Communications. Proceedings (IEEE Cat. No.02EX605), 2002. ICCSC'02, pp. 6-12.

[21] R. J. Cameron, et al, Synthesis of Network-Circuit Approach, 2rd ed. IEEE Books for Purchase : Wiley Telecom, 2018.

[22] E. Fraccaroli, et al., "Network Synthesis for Distributed Embedded Systems," IEEE Transactions on Computers, vol. 67, iss. 9, pp. 1315 - 1330. 2018.

[23] X. Liu, "Closed-Form Coverage Probability in Cellular Networks With Poisson Point Process," IEEE Transactions on Vehicular Technology, vol.68, iss. 8, pp. 8206 - 8209. 2019.

[24] J. Boiko, V. Tolubko, O. Barabash, O. Eromenko and Y. Havrylko, "Signal processing with frequency and phase shift keying modulation in telecommunications," Telkomnika(Telecommunication Computing Electronics and Control), vol. 17, no 4, pp. 2025-2038. 2019.

[25] K. S. Ahmed and S. P. Karthikeyan, "An investigation on apportion of mathematical loss in transmission loss/cost allocation approach," Indonesian Journal of Electrical Engineering and Computer Science, vol.15, no 1, pp. 1-8. 2019.

[26] L. S. Sterling, Assisting register transfer level hardware design: A Prolog Application, London: MIT Press, 2003. 